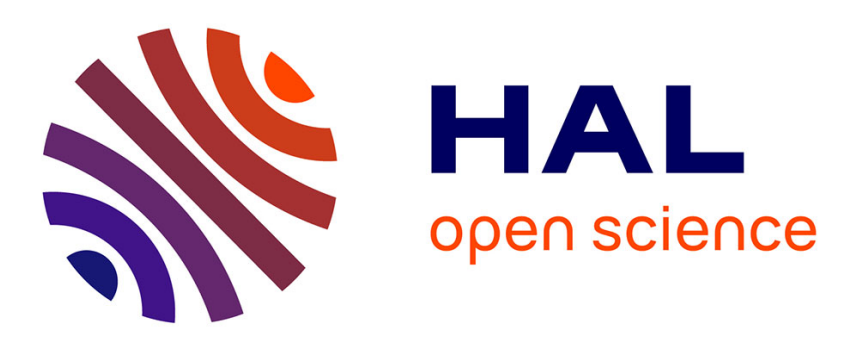

\title{
Paternité, maternité et coparentalité à l'aune de nouveaux contextes : la résidence au père et la résidence alternée
}

Agnes Martial

\section{- To cite this version:}

Agnes Martial. Paternité, maternité et coparentalité à l'aune de nouveaux contextes: la résidence au père et la résidence alternée. Dialogue. Recherches cliniques et sociologiques sur le couple et la famille, 2013, 201, pp.57-68. hal-02176565

\section{HAL Id: hal-02176565 \\ https://hal.science/hal-02176565}

Submitted on 15 Jul 2019

HAL is a multi-disciplinary open access archive for the deposit and dissemination of scientific research documents, whether they are published or not. The documents may come from teaching and research institutions in France or abroad, or from public or private research centers.
L'archive ouverte pluridisciplinaire HAL, est destinée au dépôt et à la diffusion de documents scientifiques de niveau recherche, publiés ou non, émanant des établissements d'enseignement et de recherche français ou étrangers, des laboratoires publics ou privés. 


\title{
Paternité, maternité et coparentalité à l'aune de nouveaux contextes : la résidence au père et la résidence alternée
}

\author{
Agnès Martial ${ }^{1}$ \\ In « Paternité, maternité et coparentalité à l'aune de nouveaux contextes : la résidence au père \\ et la résidence alternée $»$, Dialogue, 201, 2013, p. 57-68.
}

Dans le renouveau des études sur les formes actuelles de parenté et de parentalité, la dimension du genre permet de rendre compte des asymétries traversant les dynamiques familiales et d'y interroger l'évolution des statuts et des rôles parentaux. Les itinéraires familiaux contemporains, jalonnés de ruptures et de nouvelles unions, ont par exemple suscité nombre de recherches soulignant la primauté de la position maternelle ou la construction différenciée des relations beaux-parentales dans les familles recomposées. La paternité y est plus rarement envisagée par les sciences sociales et peu de travaux se sont penchés, en France, sur le devenir des relations père/enfant dans l'après-divorce. Il est vrai que la paternité fait déjà l'objet de très nombreuses analyses auxquelles il pourrait sembler inutile d'ajouter de nouveaux éléments, dans un domaine disciplinaire toutefois différent, constitué des apports de la psychanalyse et de la psychologie. En outre, parmi les multiples redéfinitions relationnelles de l'après-divorce, la paternité occupe une position moins visible et plus difficile à saisir. Aux côtés d'une maternité devenue centrale, et de ces parents «en plus » que représentent les nouveaux conjoints des parents, le père pourrait presque être considéré, dans certaines situations, comme un parent « en moins »...

C'est ce que laisse percevoir la redéfinition des prérogatives et des rôles parentaux après les ruptures conjugales, lorsque la résidence de l'enfant est fixée au domicile maternel tandis que les liens père/enfant se poursuivent en vertu d'un droit de visite et d'hébergement. On connaît les grands traits associés à cette organisation, qui demeure majoritairement appliquée au moment des divorces : une position maternelle prééminente, constituée à la fois d'importantes prérogatives et d'une très lourde charge éducative (Blöss, 1996; Cadolle, 2000), et une fragilisation préoccupante des relations père/enfant qui perdure aujourd'hui. En 2005, $40 \%$ des enfants de moins de 25 ans issus d'une union rompue ne voyaient leur père que rarement ou jamais (Chardon, Daguet, Vivas, 2008). Dans ces conditions, les recherches ont le plus souvent caractérisé le statut et le rôle des pères séparés ou divorcés en référence aux notions d'éloignement, d'absence, de moindre investissement dans la relation aux enfants (Martial, 2012). Le système du droit de visite et d'hébergement instaure ainsi du côté paternel des relations discontinues, éprouvées par l'absence de corésidence et de temps quotidien (Fine, Martial, 2012), et tend à creuser les asymétries sexuées traversant les rôles parentaux.

Dans le contexte d'une valorisation croissante du principe de coparentalité, l'émergence de nouveaux modes de résidence de l'enfant offre cependant l'occasion de réinterroger les conceptions et les usages de la paternité, ainsi que la manière dont elle s'articule à la maternité. Les données statistiques les plus récentes témoignent d'une progression lente mais continue des organisations permettant le partage d'un temps quotidien entre père et enfant(s) ainsi qu'une répartition théoriquement plus égalitaire des tâches parentales. La résidence alternée, reconnue par la loi du 4 mars 2002, s'est installée dans les usages même si elle demeure minoritaire : elle concernait en $200715 \%$ des enfants mineurs dont les parents ont

\footnotetext{
${ }^{1}$ Agnès Martial, chargée de recherches au CNRS, Centre Norbert Elias, Marseille ; agnes.martial@univ-amu.fr
} 
divorcé (Chaussebourg, Carrasco, Lermenier, 2009). Elle est plus fréquemment prononcée dans les divorces par consentement mutuel, où elle représentait en 2010 27,8 \% des décisions (Boisson, Wisnia-Vell, 2012). La résidence des enfants au domicile paternel, qui concerne moins de $10 \%$ des enfants mineurs au moment des divorces (Chaussebourg, Carrasco, Lermenier, 2009), représente une réalité plus importante dès lors que l'on tient compte des modifications ultérieures de la résidence des enfants. Ainsi, « dans les actions modificatives après divorce, la résidence principale a été attribuée au père dans $33 \%$ des cas, $10,5 \%$ des mineurs ont été accueillis en résidence alternée, les mères ne conservant la résidence principale que dans $56 \%$ des cas en $2010 »$ (Boisson, Wisnia-Weill, 2012). Comment sont décidées et motivées ces nouvelles organisations ? Quelle est leur incidence sur la répartition genrée de la prise en charge des enfants?

\section{L'enquête}

Nous tenterons d'apporter quelques éléments de réponse à ces questions à partir des récits et témoignages de 33 pères séparés ou divorcés ayant expérimenté ces formes d'organisation de la résidence de l'enfant. L'enquête ${ }^{2}$, d'abord conduite grâce à des contacts informels (6 pères), puis par l'entremise de la Caisse d'allocations familiales des Bouches-du-Rhône (27 pères), a permis de rencontrer des hommes ayant vécu ou vivant au moment de l'entretien une situation de résidence alternée ( 9 cas) ou une situation de résidence quotidienne des enfants à leur domicile (24 cas). La diversité des situations ayant été volontairement privilégiée, ces pères d'âges et d'origines culturelles variés (nés à Marseille, originaires d'autres régions françaises, migrants d'origine étrangère) étaient nantis de niveaux de qualification très différents et appartenaient à des catégories socioprofessionnelles très diversifiées (sans activité, ouvriers, employés ou cadres du secteur public ou privé, indépendants). La moitié de ces hommes a divorcé après un mariage tandis que les autres ont connu une séparation, ceci n'excluant pas l'intervention d'un juge aux affaires familiales sur l'organisation des modalités de la résidence ou de l'entretien de l'enfant.

À partir d'un tel groupe, il est nécessaire de tenir compte de la pluralité des conditions économiques et matérielles des ruptures conjugales ainsi que des variations sociales et culturelles des rapports de genre et de parenté. Cette diversité permet aussi d'interroger les frontières établies entre un modèle dominant de séparation "négociée » allié aux principes d'égalité et de coparentalité, que la majorité des travaux sur les recompositions familiales ont décrit à partir de l'étude des classes moyennes et supérieures, et un modèle de séparation plus « traditionnel» au sein des milieux modestes ou populaires, où la rigidité plus grande des normes de genre, le « surcodage social» des identités sexuées conduiraient à mettre en doute la pertinence même de la notion de coparentalité (Jamoulle, 2005 ; Neyrand, 2000).

\section{La résidence au domicile paternel}

Différents processus ont conduit les pères rencontrés à élever quotidiennement leurs enfants après un divorce ou une séparation. Deux motifs principaux se détachent, qui peuvent dans certains cas coexister. Le premier donne à voir cet arrangement comme le prolongement d'une répartition antérieure des rôles où le père occupait déjà une position de «parent

\footnotetext{
${ }^{2}$ Enquête réalisée dans le cadre d'un programme de recherche portant sur les redéfinitions de la paternité dans les nouvelles trajectoires familiales ANR-08-JCJC-0057-01 «Pères en solitaire: paternités contemporaines et nouvelles trajectoires familiales (France, Espagne) », dirigé par Agnès Martial.
} 
principal» dans la prise en charge quotidienne des enfants, du fait de la dynamique " égalitaire » présente au sein du couple parental ou/et du peu d'intérêt de la mère pour cette dimension de la parentalité. Les hommes témoignant de cette expérience appartiennent plutôt aux classes moyennes ou supérieures. Ils se disent déterminés à demeurer pères au quotidien quoiqu'il arrive et ont demandé, dès l'annonce de la rupture, que la résidence des enfants soit fixée chez eux. Ils se présentent comme les garants d'une continuité familiale bénéfique pour les enfants, la mère étant fréquemment, dans leurs récits, à l'origine de la séparation. On voit se dessiner dans certains cas une situation symétriquement inverse aux répartitions « traditionnelles » des rôles de genre.

Julien, 49 ans, et son ex-épouse sont tous deux architectes, parents de deux enfants. Au fil de leurs trajectoires professionnelles, la mère s'est investie de manière grandissante dans sa carrière professionnelle et il se consacrait plus qu'elle à l'éducation de leurs enfants, âgés de 7 et 10 ans au moment de leur séparation. "J'ai dit: "Si tu t'en vas je garde les enfants." Ce qui moi ne me changeait pas tant que ça parce que ça faisait un moment déjà que je m'en occupais au quotidien. On partait ensemble le matin, c'est la seule chose qu'on faisait ensemble, mais après elle rentrait toujours tard le soir, elle travaillait souvent le week-end; pour les vacances on ne savait pas trop si elle serait libre ou pas, donc finalement ça faisait un moment que je gérais, je dirais, tout ce qui est de l'intendance [...] j'avais envie de les voir, de passer du temps avec eux, de les voir grandir en fait. "

Yvan (47 ans, régisseur de théâtre, deux enfants) se présente également comme ayant toujours assumé la majeure partie de l'éducation de ses fils, décrivant une vie familiale dont la mère était relativement absente. "Il y avait cette présence que j'avais. La cuisine, par exemple, c'est moi qui gérais, les courses aussi... Bon, la maman s'occupait bien de l'entretien de la maison, et moi je m'inquiétais pour les vacances, pour les repas, l'école [...] C'est moi qui gérais les sorties, les week-ends de pique-nique; enfin, toute cette dynamique-là, c'est moi qui la créais ... [...] Les amener au parc, leur faire prendre l'air, faire du vélo, etc. C'est vrai que les souvenirs s'effacent, mais j'ai le sentiment d'avoir géré le poids d'être seul [...]. »"

Le second déterminant de la résidence quotidienne des enfants chez le père, tel qu'il apparaît dans le discours de ces hommes, est l'incapacité relative de la mère à prendre en charge les enfants. Le registre de la défaillance maternelle affleure alors dans les récits. Quel que soit leur milieu d'origine, et bien que les motifs et critères de leurs jugements varient grandement, nombre de pères s'accordent à constater que les mères de leurs enfants ne sont pas - ou pas tout à fait - de «bonnes » mères. Dans les cas les plus ordinaires, la maternité est décrite comme peu investie, caractérisée par diverses formes d'incompétence domestique ou éducative. Les mères sont parfois présentées comme peu fiables, en raison de leur inconstance amoureuse et sexuelle, de leur instabilité professionnelle ou/et d'une grande fragilité psychologique. Dans l'histoire de certaines séparations, la mère s'était vu confier la résidence habituelle lors de la rupture, ou devait accueillir les enfants en résidence alternée, mais cette solution n'a pas duré pour des raisons économiques et matérielles (perte du logement), de santé (dépression, hospitalisation), ou parce qu'un conflit mère/enfant a conduit au changement de résidence de ce dernier. Dans quelques situations, la mère s'est éloignée ou a disparu, quittant à la fois les enfants et le père. Dans deux cas, les enfants dont la résidence avait été fixée au domicile maternel ont été placés en raison de graves défaillances éducatives liées aux difficultés personnelles de la mère (addictions, délinquance, prostitution), le père récupérant finalement la « garde » des enfants.

Au fil des récits se dessinent ainsi des paternités construites en miroir d'une maternité fragile, 
affaiblie, parfois désertée. Certains de ces portraits de mères sont associés à de très lourdes problématiques sociales et psychologiques et singularisent la paternité "solitaire » comme solution ultime de prise en charge des enfants. Au-delà de ces cas particuliers, le caractère transversal d'un discours négatif à l'égard des mères au sein de ce groupe d'hommes témoigne surtout de la puissance des normes qui définissent la maternité et sanctionnent les conduites qui s'en écartent. Ces normes retentissent également dans l'inquiétude que ces hommes expriment fréquemment au sujet du bien-être de leurs enfants, craignant de ne pouvoir compenser l'éloignement de la mère. Ces pères remettent rarement en cause "l'évidence maternelle » justifiant dans la majorité des cas la résidence au domicile de la mère [Bessière et Gollac, 2010]. Ils pallient son absence, de manière plus ou moins contrainte. Ceci n'en transforme pas moins leur manière d'agir et de se penser comme pères et modifie les dynamiques articulant paternité et maternité dans l'après-rupture.

\section{Des familles « patricentrées »}

Sur bien des points, l'expérience masculine et solitaire de la parentalité fait écho à celle des familles monoparentales féminines. Dès lors que les enfants viennent vivre chez leurs pères, le temps et l'espace sont saturés d'enfants, de travail domestique et de tâches éducatives qu'il faut concilier avec le maintien d'un emploi assurant un revenu plus ou moins élevé, pour lesquels il faut aussi parfois renoncer à une vie sociale qui s'amenuise ou disparait. Les inégalités socio-économiques sont ici très présentes, la charge reposant sur le père étant d'autant plus lourde que l'on descend dans l'échelle sociale. Pour certains hommes très peu qualifiés et mal insérés sur le marché du travail, concilier un emploi et la présence de jeunes enfants au domicile est aussi difficile que pour les femmes. Presque tous les pères rencontrés ont dû adapter leur vie professionnelle aux nouvelles contraintes familiales, ce qui semble plus aisé pour les cadres, les ingénieurs et certains indépendants que pour les petits employés ou les hommes travaillant grâce à des contrats à durée déterminée, dont ils ne peuvent négocier les horaires, souvent «atypiques » et inconciliables avec la présence au foyer de jeunes enfants. Si certains pères sont en mesure de financer la garde des enfants à domicile, d'autres dépendent entièrement des structures institutionnelles de prise en charge de la petite enfance. Ils renoncent parfois à l'emploi et perçoivent le RSA en attendant que les enfants grandissent.

Se pose également la question de la contribution de l'autre parent à l'éducation et à l'entretien de l'enfant: lorsqu'elles en ont les moyens et qu'une résidence habituelle au domicile paternel a été décidée, les mères versent une pension au père - ce qui advient dans les classes moyennes et supérieures, jamais dans les milieux modestes. Il faut enfin souligner l'importance du soutien familial, très inégal selon les situations : là où certains pères bénéficient d'une aide et d'une présence très régulière assurée par les grands-parents ou par une sœur demeurée célibataire, d'autres, notamment les plus précaires et les migrants, demeurent très seuls.

L'investissement de la paternité au quotidien se traduit cependant par des gestes nouveaux, au fil de la vie domestique et matérielle, par des préoccupations éducatives envahissantes et par des échanges plus intenses avec les enfants. Ces pères sont concernés par le suivi scolaire et les relations à l'école, par la santé, l'organisation des loisirs, des vacances, etc. Ils expriment leur angoisse et leur sentiment de solitude lorsque leurs enfants, surtout à l'adolescence, rencontrent différentes difficultés scolaires, sociales et psychologiques. Mais ils commentent également l'intensité de leur relation et leur plaisir à partager avec eux la vie de tous les jours. Daniel, ouvrier, élève seul ses deux enfants adolescents : "Ça c'est fatiguant, surtout 
en travaillant : faut rentrer le soir, leur faire à manger ... Y'a plus de boulot mais je les ai. Je suis content, hein : quand je rentre le soir ils me sautent au cou, ils sont contents de me voir...

La présence de la mère et son éventuelle intervention dans l'éducation de l'enfant sont rarement mentionnées, si ce n'est pour évoquer différents conflits et désaccords concernant l'éducation de l'enfant - ou pour signaler son incompétence. Le discours de ces hommes valorise finalement la dimension solitaire de leur paternité. À cette charge parentale répond en effet une forme de prééminence éducative, plus ou moins explicitement affirmée. Une gamme étendue déploie différentes interprétations d'une coparentalité plus ou moins réalisée, allant de «C'est moi qui décide seul », en passant par « Je l'appelle et je la préviens toujours, mais c'est moi qui tranche », à " Nous en discutons toujours ensemble avant de prendre une décision " selon les situations, la capacité de la mère à participer aux décisions et la qualité des relations entre les parents.

Au-delà des décisions relatives aux grandes orientations de la vie de l'enfant, cette prééminence se lit dans différents domaines de l'éducation, à travers certaines manières de faire ou de dire, qui laissent deviner une position d'autorité ou de responsabilité dominante au sein du duo parental. Medhi, 38 ans, est arrivé d'Algérie il y a dix ans. Il est employé en CDD et à temps partiel dans une entreprise de nettoyage. Marié puis divorcé d'avec une jeune femme française, il s'est rapidement occupé de son fils ( 9 ans) au quotidien, son ex-épouse ne parvenant pas à assumer le système d'alternance initialement fixé. L'enfant se rend néanmoins en visite chez sa mère de temps en temps. Medhi veille alors au bon déroulement de ces séjours en donnant à la mère une liste de recommandations : heure du coucher, horaires de télévision, etc., qu'elle est censée observer. "Je lui fais même une liste pour sa mère [...] Ben oui! Qu'est-ce qu'il faut faire, qu'est-ce qu'il ne faut pas faire. [...] Et après quand il est revenu, je le piège. "Hier chez Maman tu as vu la télé jusqu'à quelle heure "? Il m'a dit: "J'ai vu la télé jusqu'à 9 heures et j'ai vu la télé le matin." Après je téléphone à sa mère et je dit que cela ne se fait pas: "Là, tu me casses tout le travail !" " Medhi se préoccupe également de la tenue de l'enfant lorsqu'il est chez sa mère : "Chaque année j'achète un peu plus et il le ramène chez sa mère, comme ça il se change. J'aime quand il est bien habillé, voilà. Elle ne sait pas marier les choses, par exemple la veste avec le pantalon, elle l'envoie [à l'école] n'importe comment, je n'aime pas ça. Ce n'est pas de la marque, mais bien habillé, propre. Et bien assorti. »

D'autres pères suggèrent à la mère différentes initiatives censées entretenir ou améliorer sa relation à l'enfant. Yvan, qui élève les enfants au quotidien depuis six ans, propose parfois à son ex de payer de nouvelles tenues à leurs fils plutôt que de lui verser la pension mensuelle, parce qu'il pense que «c'est bien aussi que leur maman leur achète des vêtements ». Julien raconte qu'il a "prévenu » la mère quand leur fille aînée a été hospitalisée et lui a "suggéré » de venir passer une nuit avec elle : "Je voulais tout simplement associer leur mère au fait que Laura soit à l'hôpital et ne pas être le seul. J'aurais pu techniquement le faire, mais je me disais que c'était aussi la place de leur mère d'être à leur côté à ce moment-là. » De manière plus ou moins explicite, ces pères jouent ainsi un rôle dans la définition même de la relation de la mère à l'enfant; ils se présentent alors comme des médiateurs, rôle que l'on attribue habituellement aux femmes vis-à-vis de la relation paternelle, rôle qui les valorise et les situe clairement dans la position de parent principal.

Inscrites dans la quotidienneté, ces formes de paternité mettent donc à l'épreuve les définitions traditionnelles des rôles de genre et les conceptions que les hommes se font de leur 
rôle parental. Cette nouvelle place se décline de deux manières. "Je suis le père, je suis la mère »: c'est ce qu'expriment souvent les pères les plus âgés, contraints, disent-ils, d'assumer deux rôles bien distincts afin de pallier l'absence d'une mère plus ou moins disparue de la vie des enfants. D'autres pères se réfèrent à un modèle de parentalité entendu comme un ensemble de compétences qui ne sont plus nécessairement assignées à un sexe : «En tant qu'homme, je peux assumer des tâches et des gestes habituellement réservés aux femmes et aux mères, tout en jouant mon rôle de père. »

\section{Le coût de la paternité quotidienne : pas ou peu de nouvelles unions ?}

La prééminence masculine dans la dynamique de la coparentalité induit cependant un coût particulièrement apparent dans ce groupe de pères ${ }^{3}$, qui déroge aux tendances statistiques selon lesquelles les hommes se remettraient en couple plus vite et plus fréquemment que les femmes. Certains n'ont aucune vie amoureuse, se sentant trop isolés, indisponibles, dans une situation qui ne leur permet pas de rencontrer un nouveau partenaire. D'autres conjuguent un état de célibat à quelques aventures, voire une relation régulière, mais hors de la vie familiale, comme si leurs enfants et leurs amours devaient constituer deux univers affectifs distincts et plus ou moins cachés l'un à l'autre. Quelques-uns décrivent quelques tentatives insatisfaisantes de vie commune, déçus par un premier essai qui a créé, disent-ils, des complications avec les enfants : tant que ces derniers ne sont pas autonomes, la relation paternelle passe avant la relation conjugale. Cet état de célibat suscite parfois un discours sacrificiel : "Les enfants passent d'abord, moi après », "C'est fini pour moi, maintenant c'est tout pour mes enfants », "J'ai tout donné pour eux pendant six ans ».

\section{La résidence en alternance : plus d'espace pour chacun?}

La résidence alternée se rencontre dans des situations différentes. Elle assure tout d'abord le partage d'un temps et d'un espace quotidiens à des hommes qui ne concevaient pas, disent-ils, de vivre sans leurs enfants, sans que la maternité soit pour autant disqualifiée. Le choix de la résidence alternée est par ailleurs observé dans tous les milieux rencontrés, mais cette organisation paraît plus fragile et moins pérenne dans les familles les plus modestes (dans cette enquête, basée sur l'étude de situations paternelles, c'est la mère qui échoue à assumer régulièrement la charge de l'enfant). Elle donne lieu à différentes répartitions des rôles et des prérogatives entre les parents.

Vincent, fonctionnaire territorial, 43 ans, père de trois filles (18, 15 et 10 ans), n'est pas encore divorcé. Sa femme, éducatrice, l'a quitté il y a deux ans pour vivre une nouvelle relation. Les deux ex-conjoints vivent à proximité l'un de l'autre, ce qui permet de décliner différentes formes d'alternance : les grandes changent de domicile tous les ans (l'aînée vit actuellement chez son père, la cadette chez sa mère), la benjamine change tous les quinze jours. La coparentalité s'actualise ici selon plusieurs modalités, et d'abord par la solidarité financière. Vincent habite encore l'appartement acheté par le couple peu avant la rupture, logement assez grand pour y accueillir les 3 filles, et la mère contribue toujours, dans une part réduite, au remboursement du crédit immobilier. La prise en charge des enfants au quotidien n'est pas organisée en fonction des semaines d'alternance mais selon les disponibilités des parents : la mère récupère leur plus jeune fille chaque fin de journée à la sortie de l'école, $\mathrm{y}$ compris quand l'enfant est chez son père, qui vient la chercher au domicile maternel en

\footnotetext{
${ }^{3}$ Il importe bien sûr de tenir compte du biais induit par la prise de contact, ces pères étant majoritairement déclarés auprès de la Caisse d'allocation familiale comme vivant seuls avec leurs enfants.
} 
quittant son travail. Elle la garde également chaque mercredi, que l'enfant soit ou non chez son père, et prend les enfants plus longtemps pendant les vacances car elle bénéficie de congés plus longs que ceux de Vincent. Ils essaient d'aller ensemble aux réunions scolaires et parents/professeurs, passent ensemble les fêtes familiales dédiées aux enfants (Noël, anniversaires), se téléphonent chaque soir pour parler des enfants. Comme le dit un autre père, Jean-Paul (journaliste, 49 ans, deux enfants), la résidence alternée, dans un contexte pacifié, favorise parfois une coopération parentale plus grande que dans le couple uni, du fait, semblet-il, de l'apaisement des conflits et d'un engagement plus important du père. "On ne se voit pas hormis tous les quinze jours quand on fait les changements, mais on s'appelle au téléphone, on parle assez régulièrement de leur éducation [...] C'est marrant parce que cette harmonie, qu'on n'a jamais eue quand on vivait ensemble, on l'a aujourd'hui pour l'éducation. Et je sens aussi que de sa part justement il y a un effort aussi pour éviter des malentendus... De ma part aussi... Il y a une vraie association pour ce qui est de l'éducation. $»$

Ce tableau positif ne doit cependant pas occulter une réalité plus complexe. La résidence alternée ne se déroule pas toujours dans un climat serein de coopération parentale et peut aussi prendre place dans un contexte très conflictuel, dérogeant au principe du «bon » divorce négocié et consensuel. Hervé (enseignant, père de deux enfants) qualifie de «cauchemar judiciaire » les différentes procédures qui, depuis quatorze ans, l'opposent à son ex-épouse, mêlant litiges d'ordre économique et conflits sur la résidence des enfants. Se décrivant comme un père très investi, il s'est battu pour obtenir qu'une résidence alternée (transformée depuis peu en résidence principale à son domicile) soit instaurée. Mais l'intensité du conflit fut telle que tout échange parental lié à l'éducation des enfants était réinterprété à la lumière de la rivalité père/mère, réactivant un interminable combat où chacun tentait de disqualifier l'autre parent. Ici, la revendication égalitaire portée par Hervé s'est muée en relation de concurrence entre paternité et maternité.

La résidence alternée n'est pas non plus nécessairement synonyme d'égalité des partages parentaux. L'écart entre le modèle idéal qu'elle est censée incarner et la réalité des pratiques est souligné par différentes études, car les asymétries traversant la répartition des rôles parentaux dans les couples unis sont fréquemment reconduites au-delà de la rupture conjugale, malgré l'équilibre que la nouvelle organisation des relations parents-enfants pourrait théoriquement instaurer. La mère continue alors d'assumer la plus grande part des responsabilités et des tâches éducatives ou d'aménager des temps de présence plus importants auprès des enfants dans les processus de conciliation entre famille et emploi (Brunet, Kertuldo, Malsan, 2008 ; Cadolle, 2008, 2011). Sylvie Cadolle distingue ainsi les couples « égalitaires » qui parviennent à équilibrer les dimensions matérielles et éducatives des relations de chaque parent à l'enfant, les couples « traditionnels rénovés » qui assument des formes d'arrangements reconduisant un modèle " père pourvoyeur/mère éducatrice » tout en assurant au père le partage d'un temps quotidien avec ses enfants, et les couples qui s'affrontent, entre pères convaincus des avantages de la résidence alternée et mères exprimant de forts sentiments d'injustice et d'insatisfaction.

\section{Conclusion}

Ce trop court aperçu des situations nouvelles d'exercice des rôles paternels permet de souligner l'importance des contextes résidentiels pour la redéfinition des rapports de genre au sein des familles recomposées et les variabilités sociales de leurs interprétations. Résidence au domicile paternel comme résidence alternée traduisent l'existence d'une reconnaissance et 
d'une valorisation encore fragiles mais grandissantes de la "paternité quotidienne » au sein des familles dissociées et recomposées, à travers de nouveaux usages qui ne sont pas l'apanage des seules classes moyennes et supérieures. Toutefois, elles recèlent des implications différentes selon les appartenances sociales et ne font pas l'objet des mêmes interprétations au regard de la notion de coparentalité. En témoignent la plus grande fragilité des situations de résidence alternée dans les milieux sociaux les moins dotés et la disqualification de la maternité dans les situations de résidence chez le père au sein des milieux les plus modestes, qui conduisent à des formes de monoparentalité masculine confrontée aux mêmes difficultés que les foyers de mères seules. Dans les milieux plus favorisés, la résidence en alternance n'équilibre pas d'un seul coup, par le seul effet de l'égalité du temps partagé, des relations de genre en lente recomposition, mais ouvre de nouveaux espaces aux redéfinitions conjointes des rôles paternels et maternels.

\section{Bibliographie}

Bessière, C. ; Gollac, S. 2010. «Au tribunal des couples. Situations professionnelles des conjoints et procédures judiciaires de séparation conjugale », Rapport de recherche pour la Mission Droit et Justice, Paris.

Blöss, T. 1996. Éducation familiale et beau-parenté: l'empreinte des trajectoires biographiques, Paris, L'Harmattan.

Brunet, F. ; Kertuldo, P. ; Malsan, S. 2008. Étude sociologique de la résidence en alternance de parents séparés, Fors Recherche sociale, Dossier d'Étude de la CNAF, 109.

Boisson, M. ; Wisnia-Weill, V. 2012. «Face à la désunion du couple, comment favoriser l'implication des pères auprès de leurs enfants ? Perspectives internationales ", Note du Centre d'analyse stratégique, Département Questions Sociales.

Cadolle, S. 2000. Etre parent, être beau-parent, Paris, Odile Jacob.

Cadolle, S. 2005. «C'est quand même mon père! La solidarité entre père divorcé, famille paternelle et enfants adultes », Terrain, 45, 83-96.

Cadolle, S. 2008. «La résidence alternée : ce qu'en disent les mères », Informations sociales, $149,68-81$.

Chardon, O. ; Daguet, F. ; Vivas, E. 2008. «Les familles monoparentales », Insee Première, 1195.

Chaussebourg, L. ; Carrasco, V. ; Lermenier, A. 2009. Le divorce, Rapport pour le Secrétariat général, sous-direction de la statistique, des études et de la documentation, ministère de la Justice.

Fine, A.; Martial, A. 2012. "Anthropologie et roman. À propos des pères divorcés ", Ethnologie française, 1, vol. 42, 155-164.

Jamoulle, P. 2005. Des hommes sur le fil. La construction de l'identité masculine en milieu précaire, Paris, La Découverte.

Martial, A. 2003. S'apparenter. Ethnologie des liens de familles recomposées, Paris, Éditions de la Maison des sciences de l'homme.

Martial, A. ; 2005. « Comment rester liés ? Les comptes des familles recomposées », Terrain, 45, 67-82.

Martial, A. 2012. " Paternités contemporaines et nouvelles trajectoires familiales ", Ethnologie française, 1, vol. 42, 105-116.

Martial, A. 2009. «Le travail parental : du côté des pères séparés et divorcés », Informations sociales, 4, 54, 96-104.

Neyrand, G. 2000. L'enfant, la mère et la question du père, Paris, PUF.

\section{Résumé}


À partir d'une enquête par entretiens menée en 2010 auprès de trente-trois pères séparés ou divorcés, cet article interroge sous l'angle du genre la redéfinition des rôles parentaux, dans le contexte de la résidence quotidienne au domicile paternel et de la résidence alternée. À partir d'un groupe socialement très diversifié, il montre que ces formes d'organisation résidentielle conduisent dans tous les milieux à la reformulation de la paternité. Ces situations font cependant l'objet d'interprétations socialement différenciées au regard de la notion de coparentalité, construisant d'un côté une paternité solitaire associée à la disqualification de la maternité, de l'autre une relation plus égalitaire, actualisable dans la résidence alternée.

\title{
Mots-clés
}

Maternité, paternité, séparation conjugale, résidence alternée, pères isolés.

Fatherhood, motherhood and shared parenting in new residential contexts (residence at father's home, shared residence)

\begin{abstract}
Based on 33 interviews with separated and divorced fathers, realised in 2010, this article interrogates the gendered redefinitions of the parental roles, in the context of the daily residence in father's house, and in the context of shared residence. From a very socially diversified group, it shows that these forms of residential organization lead in all social classes to the reformulation of fatherhood. However, these situations are differently related to the notion of "shared parenting" showing on one side a solitary fatherhood associated with a disqualified motherhood, and on the other a more egalitarian relation, occurring more often in shared residence.
\end{abstract}

